\title{
Estudo de alguns parâmetros básicos da soldagem GMAW com transferência por curto-circuito
}

\author{
(Evaluation of Basic Parameters of Short-Circuit GMA Welding)
}

\author{
Paulo J. Modenesi ${ }^{1}$, Maria Celeste Monteiro de Souza Costa², Ivan J. Santana ${ }^{2}$, João Paulo Pereira Berganholi ${ }^{3}$ \\ ${ }^{1}$ Depto. Engenharia Metalúrgica e de Materiais, UFMG, Belo Horizonte, MG, Brasil, modenesi@demet.ufmg.br \\ ${ }^{2}$ Depto. Engenharia de Materiais, CEFET-MG, Belo Horizonte, MG, Brasil \\ ${ }^{3}$ Curso de Graduação em Engenharia Metalúrgica, UFMG, Belo Horizonte, MG, Brasil
}

\begin{abstract}
Resumo
O processo de soldagem GMAW é caracterizado por uma forte interdependência de seus parâmetros operacionais e uma grande sensibilidade a variações destes. Estas características podem causar variações nas condições operacionais, dificultando o estabelecimento e a reprodução destas condições que, usualmente precisam ser verificadas pela realização de testes. Como uma forma de minimizar esta dificuldade e facilitar o entendimento da relação entre as variáveis deste processo, tem-se trabalhado no desenvolvimento de um modelo matemático capaz de simular aspectos elétricos e operacionais da soldagem GMAW. O objetivo do presente trabalho foi estudar procedimentos de determinação e fatores que afetam duas variáveis fundamentais para o desenvolvimento deste modelo (a resistividade elétrica do arame durante a soldagem e a soma das quedas de tensão anódica e catódica no arco, $U_{A C}$ ) na soldagem com transferência por curto-circuito. Os testes experimentais envolveram a deposição mecanizada de cordões emaço de baixo carbono usando arames do mesmo material com diferentes gases de proteção. Metodologias para a determinação dos parâmetros citados foram desenvolvidas com base em procedimentos similares indicados na literatura. Os detalhes destes procedimentos são avaliados, os resultados obtidos são comparados com dados da literatura e métodos para estimar a resistência elétrica entre o bico de contato e o eletrodo são apresentados. Os resultados sugerem valores para esta resistência entre 6 e $9 \mathrm{~m} \Omega$.
\end{abstract}

Palavras-chave: MIG/MAG; Arco elétrico; Curto-circuito; Modelagem.

\begin{abstract}
GMA welding is characterized by a strong dependence between its operational parameters and a high sensitivity to small variations of those. These characteristics tend to make it harder to replicate welding conditions. It is also a barrier to welding automation and makes it necessary to perform a number of real welding tests before determining operational conditions for a given application. In order to contribute to reduce these limitations of GMA welding, the authors of the present work have been working on the development of a mathematical model to simulate the variation of operational and electrical parameters during welding. This paper aims to evaluate methodologies to determine two key variables of this model (the electrode electrical resistivity and the sum of the voltage drops in the arc anode and cathode regions) in short-circuit welding. Experimental trials were performed in a mechanized jig by depositing bead-onplate welds on low-carbon steel bars. Methodologies to evaluate those variables were developed based in proposals from the literature. Experimental details of these methodologies are analyzed and their results are compared to results from the literature. These procedures are evaluated, compared with data from the literature and new procedures are proposed to estimate those parameters and the contact resistance between the contact tip and the electrode. Results suggest values between 6 and $9 \Omega$ for this last parameter.
\end{abstract}

Key-words: GMAW; Electric arc; Short circuit transfer; Modeling.

\section{Introdução:}

O processo de soldagem GMAW é um dos mais utilizados industrialmente. Isto se deve principalmente a sua versatilidade, ao excelente nível de produtividade, confiança, facilidade de utilização e automatização. Porém, o processo oferece certa dificuldade associada à definição correta dos parâmetros de soldagem, principalmente devido ao número relativamente alto de variáveis e, sobretudo, a uma forte inter-relação entre

(Recebido em 11/11/2010; Texto final em 23/02/2011).

Artigo originalmente publicado no CONSOLDA 2010,
estas.É possível determinar parâmetros de soldagem adequados para uma determinada situação por meio de testes, porém este procedimento geralmente consome recursos e tempo excessivo. Portanto, a criação de ferramentas capazes de prever e ou controlar de forma confiável as variáveis do processo possibilitaria a melhoria da qualidade do produto final, a segurança operacional, a diminuição do custo de fabricação e o melhor entendimento da influência de cada variável.

Nos últimos 20 anos, vários modelos matemáticos foram desenvolvidos para descrever aspectos físicos e operacionais do processo GMAW. Embora o processo esteja bem estabelecido, a complexidade dos fenômenos envolvidos na interação entre metal líquido e sólido, gases de proteção e arco elétrico e as características e interações entre as variáveis do processo e os 
modos de transferência e outros carecem ainda de um melhor entendimento. Para auxiliar no entendimento de alguns destes aspectos, modelos enfocando diferentes aspectos deste processo têm sido desenvolvidos. Entre estes, se incluem modelos essencialmente empíricos ou de base teórica para prever, por exemplo, o formato do cordão [1], para estudar características do arco elétricoe da transferência de metal [2], da poça de fusão [3] e dos aspectos elétricos e operacionais do processo [4]. Nesta última classe, destacam-se, por exemplo, modelos baseados na equação do circuito elétrico de soldagem e do balanço de massa no eletrodo:

$$
\begin{aligned}
& \frac{d l}{d t}=\frac{1}{L}\left(U_{\text {Fonte }}-\sum U_{i}\right) \\
& \frac{d l_{a}}{d t}=w-f
\end{aligned}
$$

Onde $U_{\text {Fonte }}$ é a tensão da fonte, $\Sigma U_{i}$ é a soma das diferentes quedas de tensão ao longo do circuito de soldagem (incluindo, por exemplo, as quedas de tensão no eletrodo, no contato entre o eletrodo e o bico e no arco), $w$ e $f$ são, respectivamente, as velocidades de fusão e de alimentação do eletrodo, $L$ é a sua indutância, $I$ é a corrente de soldagem e $l_{a}$ é o comprimento do arco. Entre as quedas de tensão, $U_{i}$, duas são particularmente importantes: a queda ao longo do eletrodo $\left(U_{e}\right)$ e a do arco elétrico $\left(U_{a}\right)$. Estas podem ser representadas como:

$$
\begin{aligned}
& U_{e}=\rho \frac{s}{A} I, \mathrm{e} \\
& U_{a}=U_{A}+U_{C}+E l_{a}=U_{A C}+E l_{a}
\end{aligned}
$$

Onde $\rho$ é a resistividade elétrica média do eletrodo, $s$ e $A$ são o seu comprimento e área da seção transversal, respectivamente, $U_{A}$ e $U_{C}$ são as quedas de tensão anódica e catódica e $E$ é o campo elétrico médio da coluna do arco.

O presente trabalho se insere em um projeto que propõe o desenvolvimento de modelos matemáticos para simular aspectos operacionais e elétricosdo processo GMAW usando o sistema de equações indicado acima. Uma parte essencial deste desenvolvimento é a representação das diferentes quedas de tensão no circuito e de seu comportamento durante a soldagem, o que pode ser feito seja através de medidas experimentais seja através de modelagem, o que determina essencialmente as características e potencialidades do modelo. Neste contexto, o trabalho descreve, aplica e avalia metodologias para a determinação, em testes com transferência por curto-circuito, de medidas da resistividade elétrica do arame-eletrodoe da soma das quedas de tensão anódica e catódica no $\operatorname{arco}\left(U_{A C}\right)$.

\section{Considerações Gerais}

Durante a soldagem GMAW, o eletrodo é aquecido desde uma temperatura inicial, próxima da temperatura ambiente, até a sua fusão e destacamento, na forma de gotas de metal líquido, a uma temperatura que, no caso de arames de aço, pode ser superior a $2000^{\circ} \mathrm{C}$. O aquecimento do eletrodo ocorre de duas formas principais: pelo arco elétrico e por efeito Joule. Adicionalmente, as condições de soldagem, particularmente, os valores de corrente e tensão de operação e o modo de transferência de metal, que dependem dos ajustes do equipamento, dos insumos usados e de considerações geométricas, são influenciadas pelas variações de tensão que ocorrem ao longo do circuito elétrico de soldagem (figura 1). Assim, o conhecimento das quedas de tensão existentes ao longo do circuito de soldagem e de sua dependência das diferentes variáveis do processo é importante para uma melhor compreensão de diferentes aspectos do processo e para o desenvolvimento de modelos do mesmo que simulem, por exemplo, o seu comportamento elétrico. Entre estas quedas, o presente trabalho se concentrará nas associadas com a resistência elétrica do eletrodo e em uma parte da resistência do arco (isto é, aquelas devidas às suas regiões de transição entre o eletrodo e entre a peça).

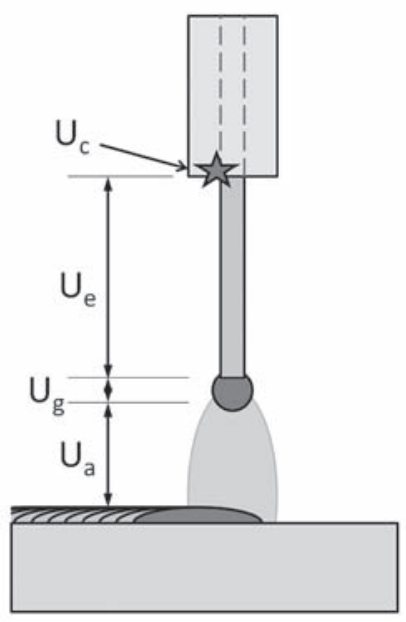

(a)

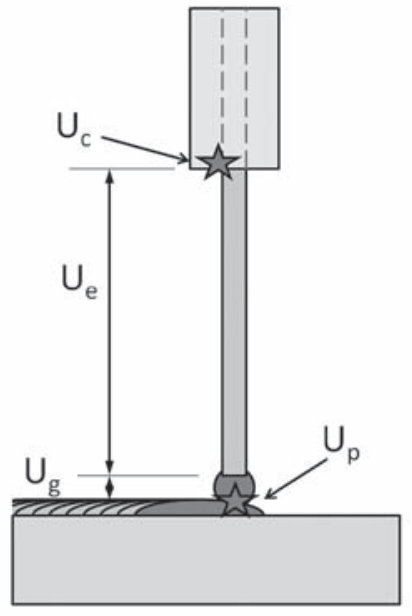

(b)
Figura 1. Componentes responsáveis pela tensão medida entre o bico de contato e a peça durante a soldagem em condições de (a) arco e de (b) curto-circuito. $U_{c}$ - tensão de contato bico/

eletrodo, $U_{e}$ - queda de tensão no eletrodo, $U_{g}-$ queda de tensão na gota de metal líquido, $U_{a}$ - queda de tensão no arco e $U_{p}$ - tensão de contato entre a gota de metal e a poça de fusão.

\subsection{Resistividade elétrica do eletrodo $\left(\rho_{e}\right)$ :}

A resistência elétrica $(R)$ de um fio metálico pode ser determinada a partir da resistividade do material $(\rho)$ e do comprimento $(L)$ e da área de sua seção transversal $(A)$ :

$$
R=\rho \cdot L / A R=\rho \cdot L / A
$$

A resistividade de uma liga metálica tende, em geral, a aumentar com o aumento de seu teor de elementos de liga (ou impurezas), de seu nível de encruamento e da temperatura [56]. De acordo com o HandbookofChemistryandPhysics [7], a resistividade do ferro puro a $20^{\circ} \mathrm{C}$ é $0,0961 \mu \mathrm{Ohm}$.m. A figura 2 mostra a variação da resistividade em função da temperatura paradiferentes ligas metálicas [8].

$\mathrm{Na}$ soldagem GMAW, a densidade de corrente no eletrodo é relativamente elevada $\left(100-300 \mathrm{~A} / \mathrm{mm}^{2}\right)$ e, portanto, 
o aquecimento do eletrodo por efeito Joule pode contribuir de forma importante para a sua fusão. $\mathrm{O}$ cálculo direto desta contribuição pode ser difícil porque a temperatura (e, desta forma, a resistividade do eletrodo) varia continuamente de uma temperatura próxima à ambiente (junto ao bico de contato) até a sua temperatura de fusão, ou mesmo de ebulição (junto ao arco) [9].

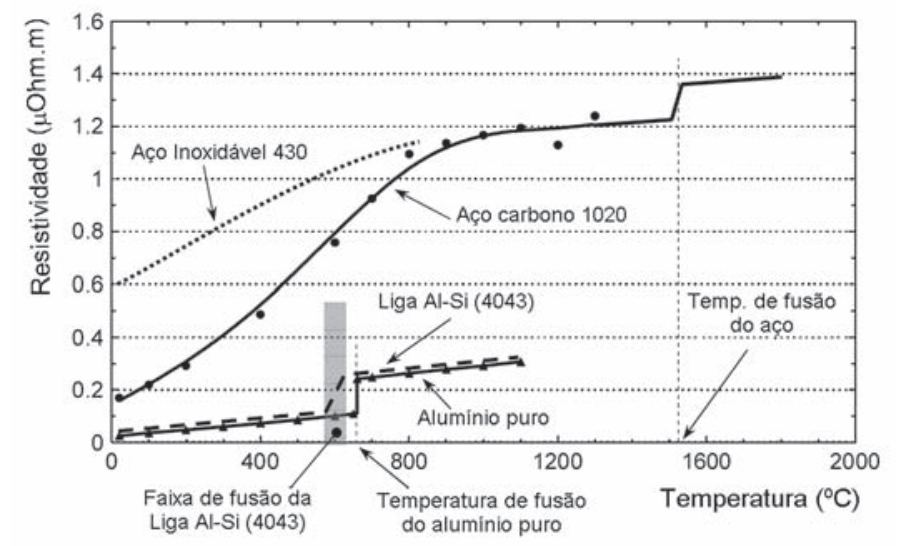

Figura 2. Variação da resistividade elétrica com a temperatura para diferentes ligas metálicas [8].

A resistividade do eletrodo, em condições reais de soldagem, pode ser estimada em condições de transferência por curto-circuito a partir da análise dos sinais de corrente e tensão de soldagem. Durante a ocorrência de um curto-circuito, a tensão $\left(\mathrm{U}_{c c}\right)$ de soldagem cai para valores abaixo de $10 \mathrm{~V}$ que, considerando esta medida ter sido feita entre o bico de contato e a peça, pode ser representada por:

$U_{c c}=U_{c}+U_{e}+U_{g}+U_{p}=\left(R_{c}+R_{e}+R_{g}+R_{p}\right) \cdot I$

Onde $R_{c}$ é a resistência de contato entre o bico e o arame, $R_{e}$ é a resistência elétrica do eletrodo, $\mathrm{R}_{\mathrm{g}}$ é a resistência da gota, $R_{p}$ é a resistência de contato entre o eletrodo e a poça de fusão e $I$ é a corrente.

Valores, na literatura, de $R_{c}$ são muito escassos. Terasaki e Simpson [4] usaram, em uma modelagem do processo GMAW, o valor de $1,5 \mathrm{~m} \Omega$, sendo este baseado em um trabalho de Huismann [10]. Vilarino [11] menciona valores em torno de $3,4 \mathrm{~m} \Omega$ e Ersoy e Colaboradores [12] mediram, usando o método de quatro contatos, valores entre 14 e $60 \mathrm{~m} \Omega$ e 150 e $200 \mathrm{~m} \Omega$ para, respectivamente, bicos novos e gastos. Por outro lado, os autores do presente trabalho não encontraram referências quanto a possíveis valores de $R_{p}$. Desta forma, supondo a existência de um bom contato elétrico pelo metal líquido entre a poça e o eletrodo, esta resistência poderia ser considerada, pelo menos inicialmente, como nula. Finalmente, neste trabalho, $R_{g}$ será considerada como parte de $R_{e}$.

A partir da equação (6) e da definição de resistividade elétrica (equação 5), o valor médio desta propriedade do eletrodo, em condições de curto-circuito durante a soldagem pode ser calculado por:

$$
\rho_{\varepsilon}=R_{\varepsilon} \cdot \frac{A}{s}=\left(R_{c c}-R_{c}-R_{p}\right) \cdot \frac{A}{s}
$$

Onde $R_{c c}$ é a resistência médiade soldagem durante os períodos de curto-circuito (medida em testes experimentais, ver metodologia), $s$ é o comprimento do eletrodo (suposto como igual à distância do bico de contato à peça) e $A$ é a área da seção transversal do eletrodo. Deve-se considerar, contudo, duas situações específicas:

(a) Em soldagem, curtos-circuitos de pequena duração (em geral, inferior a $1 \mathrm{~ms}$ ) podem ocorrer sem transferência de metal [13]. Neste caso, pode-se esperar que a resistência de contato eletrodo/poça $\left(R_{p}\right)$ possa ter valores não desprezíveis.

(b) Durante o curto-circuito, a corrente eleva-se fortemente, tendendo a diferir da corrente média de soldagem, particularmente de seu valor durante os períodos de arco. Desta forma, o valor de $R_{c c}$ será medido para valores variáveis de corrente que poderão ter um valor médio diferente da corrente média para os períodos em que o arco opera. Além disto, durante o curto-circuito, o eletrodo é aquecido exclusivamente por efeito Joule. Assim, a distribuição de temperatura ao longo do eletrodo deve ser alterada e o seu valor médio pode se tornar maior do que durante os períodos com o arco operando.

\subsection{Soma das quedas anódica e catódica do arco elétrico $\left(\mathrm{U}_{\mathrm{AC}}\right)$ :}

$\mathrm{Na}$ soldagem com transferência por curto-circuito, o comprimento do arco é progressivamente reduzido até se tornar nulo no momento do curto. Em uma primeira aproximação [1415], o arco elétrico pode ser considerado como sendo formado por três regiões (regiões anódica e catódica e coluna), sendo que, para condições específicas (por exemplo, mesmo gás de proteção, metal base e consumível) a tensão na coluna pode ser considerada como proporcional ao comprimento do $\operatorname{arco}\left(L_{a}\right)$. Levando em conta esta simplificação e as mesmas considerações do item anterior, a tensão de soldagem $\left(U_{\text {arc }}\right)$, enquanto o arco estiver operando, pode ser representada por:

$$
U_{a r c}=\left(R_{c}+R_{e}\right) \cdot i+U_{A C}+E \cdot L_{a}
$$

Onde $E$ é o módulo do campo elétrico médio na coluna do arco. Assim, uma estimativa de $U_{A C}$ pode ser obtida através de medidas de $U_{a r c}$ e $U_{c c}$ feitas, respectivamente, imediatamente antes e imediatamente após o início de um curto-circuito (figura 3), isto é:

$$
\begin{aligned}
& \left(U_{a r c}\right)_{0}=\lim _{L a \rightarrow 0}\left[\left(R_{c}+R_{\theta}\right) \cdot i+U_{A C}+E \cdot L_{a}\right]= \\
& \left(R_{c}+R_{\varepsilon}\right) \cdot\left(i_{\text {arc }}\right)_{f i m}+U_{A C} \\
& \left(U_{c c}\right)_{0}=\left(R_{c}+R_{e}+R_{p}\right) \cdot\left(i_{c c}\right)_{i n i}
\end{aligned}
$$

Supondo-se que as correntes no momento da extinção do arco $\left[\left(i_{a r c}\right)_{f i m}\right]$ e no início do curto $\left[\left(i_{c c}\right)_{i n i}\right]$ sejam iguais e que as resistências do eletrodo e de contato com o bico também sejam similares nestes instantes, tem-se: 


$$
\left(U_{a r c}\right)_{0}-\left(U_{c c}\right)_{0}=U_{A C}-\left(R_{p}\right) \cdot\left(i_{c c}\right)_{\text {ini }} \approx U_{A C}
$$

Isto é, esta diferença pode ser usada como uma estimativa de $U_{A C}$ desde que, além da suposição das igualdades dos valores correntes e das resistências do eletrodo e de contato com o bico, possa-se assumir que a resistência de contato entre o eletrodo e a poça seja desprezível.

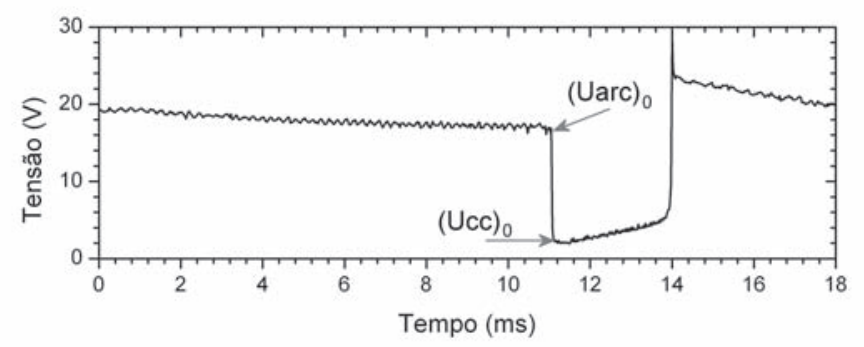

Figura 3. Definições de $\left(U_{a r c}\right)_{0}$ e $\left(U_{C C}\right)_{0}$.

Suposições similares foram adotadas por Scotti e Colaboradores [16] que, ainda, usaram a filmagem de alta velocidade (2000 quadros por segundo) sincronizada com os sinais elétricos de soldagem para observar a transferência metálica e determinar o momento mais adequado para a medição das tensões das equações acima. Os autores desenvolveram um programa computacional que identificava, nos oscilogramas de tensão e corrente, os momentos de reignição do arco e a ocorrência de cada curto-circuito além de determinar automaticamente os valores instantâneos e médios do comprimento de arco, extensão do eletrodo, diâmetro das gotas e a freqüência de transferência. A tensão de soldagem antes do curto foi tomada como um valor médio de tensão medido durante o que os autores definiram como o período de arco estável e propuseram um fator de correção para eventuais diferenças entre as correntes medidas nos períodos de arco e de curto-circuito.

\section{Metodologia}

\subsection{Testes de soldagem}

No presente trabalho, metodologias para a determinação da resistividade elétrica do eletrodo em condições de soldagem e a soma das quedas de tensão anódica e catódica do arco foram desenvolvidas e avaliadas. Para esta avaliação foram feitos testes de soldagem GMAW com arames de aço baixo carbono sobre chapas de material similar. Além disto, resultados de testes anteriores, disponíveis na biblioteca de sinais elétricos de soldagem de um dos autores, foram também usados. Os testes desenvolvidos especificamente para o presente trabalho foram feitos com arame de aço carbono (AWS ER70S6) de 1,2 mm de diâmetro e barras de aço ABNT 1010 de 9 mm de espessura.

Os testes foram realizados em um dispositivo mecanizado para a soldagem GMAW com uma fonte eletrônica chaveada no secundário. Nos testes realizados, foram variados a velocidade de alimentação $(f)$, distância do bico de contato à peça $(D B C P)$, gás de proteção $\left(\mathrm{CO}_{2}\right.$ e mistura $\left.\mathrm{Ar}-15 \% \mathrm{CO}_{2}\right)$ e a tensão de operação. A velocidade de soldagem usada $(v)$ foi proporcional à de alimentação de arame $[v=0,1 f]$ para reduzir possíveis efeitos do formato da poça de fusão nos resultados. Durante os testes, os valores de corrente e tensão de soldagem foram registrados com auxílio de um sistema digital de aquisição de dados. Foram registrados $6 \mathrm{~s}$ de teste, após um período inicial de cerca de $5 \mathrm{~s}$ para estabilização das condições operacionais, usando taxas de aquisição de dados $\left(F_{A Q}\right)$ de 10 ou de $20 \mathrm{kHz}$. Em um conjunto de testes, a taxa de aquisição de dados foi variada de 2,5 a $160 \mathrm{kHz}$.

\subsection{Processamento inicial dos dados}

Os arquivos de dados foram processados com auxílio de um programa especialmente desenvolvido para este fim (Programa Sinal). Inicialmente, este processamento envolveu:

- Cálculo dos valores médios de corrente e tensão de soldagem e do período de transferência (tempo entre o início de dois curtos-circuitos consecutivos, $T$ ), do tempo de curtocircuito $\left(T_{c c}\right)$ e do fator de curto-circuito $\left(F_{c c}=T_{c c} / T \times 100\right)$.

- Cálculo da resistência média de soldagem durante curtoscircuitos $\left(R_{c c}\right)$ : Os valores de resistência durante um teste foram calculados diretamente pela divisão ponto a ponto dos valores de corrente e tensão deste teste. Os resultados obtidos foram colocados em um gráfico de resistência em função da corrente (figura 4). $R_{c c}$ foi tomada como o valor médio das resistências menores que $40 \mathrm{mOhm}$. Este valor foi definido com base em testes anteriores como um valor intermediário entre as resistências do processo na presença do arco elétrico e em curtocircuito. Os valores de $R_{c c}$ foram usados para se obter estimativas iniciais da resistividade do eletrodo $\left(\rho_{e}\right)$ usando a equação (3) e considerando inicialmente os valores de $R_{c}$ e $R_{p}$ como nulos e $s$ como igual ao $D B C P$.

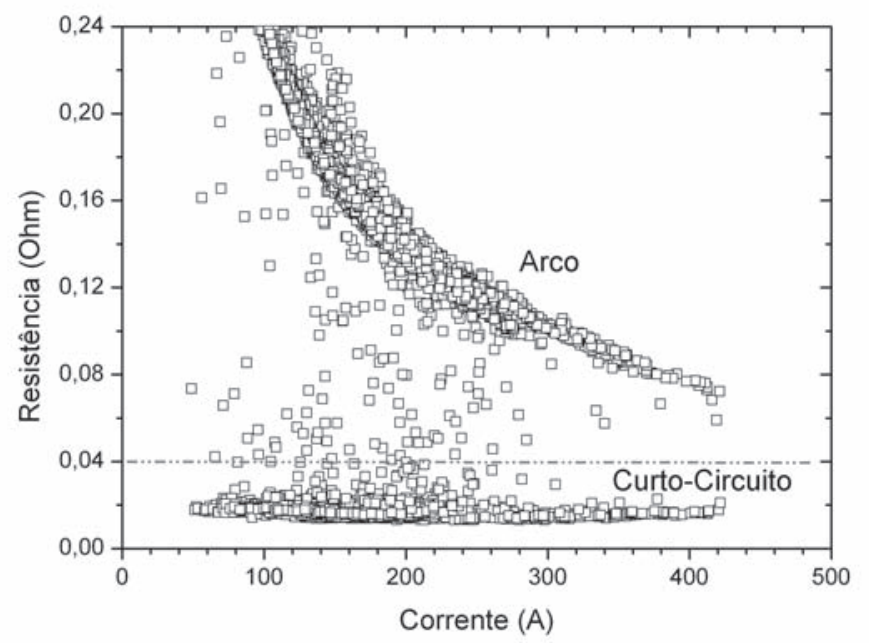

Figura 4. Procedimento para a determinação de $\left(\mathrm{R}_{\mathrm{CC}}\right)_{\text {Média }}$.

- Cálculo de $\mathrm{U}_{\mathrm{AC}}$ : Este parâmetro foi determinado com base na equação (7) usando uma rotina do programa que calcula a diferença de cada valor medido de tensão e um valor seguinte medido após um tempo igual a $N / F_{A Q}$, isto é:

$$
\Delta U(i)=U(i+N)-U(i) \Delta U(i)=U(i+N)-U(i)
$$


Neste trabalho usou-se um valor de $N$ igual a dois. Observou-se que a diferença calculada entre pontos adjacentes $(N=1)$, para as taxas de aquisição usadas, tende a fornecer estimativas menores de $U_{A C}$, pela aquisição frequente de medidas entre as condições de arco e de curto-circuito.

Uma vez obtidos as diferenças (equação 7), usou-se o programa para determinar os seus valores mínimos entre os seus picos negativos (figura 5), os quais correspondem à transição entre a condição de operação com arco para a em curto-circuito. Para este procedimento, usou-se uma rotina que permite separar os picos de um determinado vetor de dados cujo valor é inferior a um valor de corte fornecido pelo usuário. No presente trabalho, para este fim, foi usado um valor de corte de $-12 \mathrm{~V}$, escolhido após uma série de testes preliminares.

\subsection{Processamento final dos dados/avaliação}

Os dados determinados (valores de $R_{e}$ ou de $\rho_{e}$ e de $U_{A C}$ ) a partir dos testes de soldagem deste trabalho, juntamente com resultados de testes de trabalhos anteriores, foram comparados com dados de literatura. Além disto, foi feita uma análise crítica da influência de diferentes fatores nos resultados obtidos. Um melhor detalhamento desta análise é apresentado na próxima seção deste trabalho (resultados e discussão).

\section{Resultados e Discussão}

A tabela 1 mostra valores determinados de $\rho_{e}$ e de $U_{A C}$ para algumas condições de ensaio. Nesta tabela, os valores de $\rho_{e}$ foram calculados pela equação (3) considerando as resistências de contato nulas.

Os resultados da tabela 1 sugerem algumas relações entre as variáveis do processo e os valores de $\rho_{e}$ e de $U_{A C}$, com destaque do efeito do gás de proteção e da tensão de soldagem na soma das quedas anódica e catódica. Algumas destas relações serão discutidas nas seções seguintes, contudo, a ênfase neste trabalho será uma avaliação da metodologia usada e suas conseqüências.

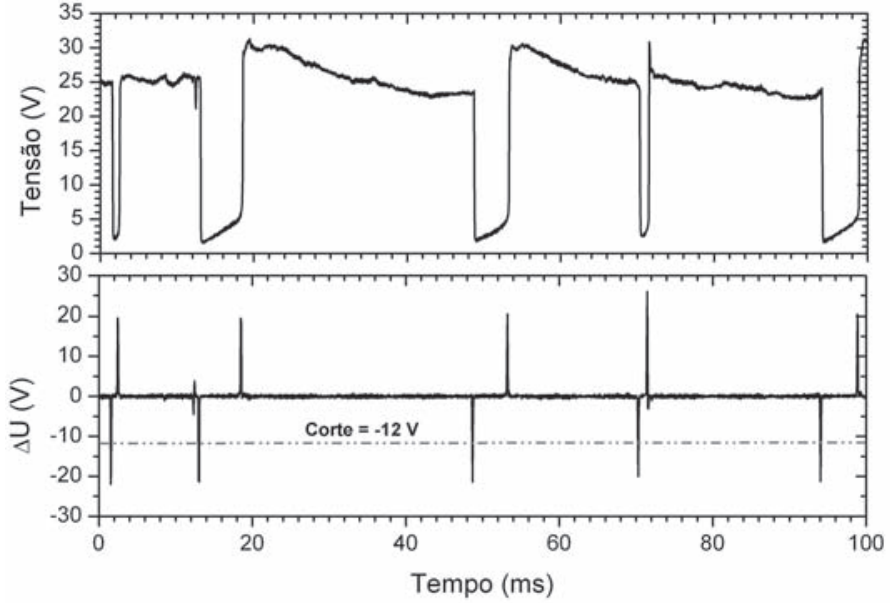

Figura 5. Procedimento para a determinação de $U_{A C}$.

\subsection{Resistência e resistividade do eletrodo}

Comparando os valores obtidos de resistividade (tabela 1) com os valores da resistividade de um aço de baixo carbono (figura 2), observa-se que estes correspondem a uma temperatura efetiva no eletrodo superior a $800^{\circ} \mathrm{C}$. Este valor é muito elevado, possivelmente irreal, considerando resultados de modelagem da distribuição da temperatura no eletrodo [17] e de análise metalográfica da ponta de eletrodos [18]. Assim, para se obter um valor de resistividade com um maior significado físico, as resistências de contato do eletrodo com o bico e a poça devem ser consideradas.

A figura 6 mostra como o valor médio do curto-circuito varia com a sua duração. Observa-se uma queda da resistência coma duração, o que inicialmente pode ser considerado como inesperado se a tendência do eletrodo se aquecer mais uniformemente durante o curto-circuito puder ser considerada como relevante. Por outro lado, este efeito pode ser associado com uma menor influência deste aquecimento em conjunto com uma maior resistência de contato com a poça durante os curtoscircuitos de menor duração.

Tabela 1. Resultados (média \pm desvio padrão) de alguns ensaios. $f$ - Velocidade de alimentação, $U o$ - Tensão ajustada na fonte, $n-$ Número de réplicas na condição, $I$ e $U$ - Corrente e tensão médias de soldagem.

\begin{tabular}{c|c|c|c|c|c|c|c|c|c}
\hline $\begin{array}{c}f \\
(\mathrm{~m} / \mathrm{min})\end{array}$ & $\begin{array}{c}U o \\
(\mathrm{~V})\end{array}$ & $\begin{array}{c}D B C P \\
(\mathrm{~mm})\end{array}$ & $\begin{array}{c}\text { Gás de } \\
\text { proteção }\end{array}$ & $n$ & $I(\mathrm{~A})$ & $U(\mathrm{~V})$ & $F_{c c}(\%)$ & $\begin{array}{c}\rho_{\mathrm{e}} \\
(\mu \Omega . \mathrm{m})\end{array}$ & $\begin{array}{c}U_{A C} \\
(\mathrm{~V})\end{array}$ \\
\hline 4 & 21 & 14 & $\mathrm{CO}_{2}$ & 2 & $178 \pm 3$ & $18,4 \pm 0,0$ & $30,9 \pm 0,3$ & $1,33 \pm 0,02$ & $20,6 \pm 0,1$ \\
\hline 4 & 21 & 18 & $\mathrm{CO}_{2}$ & 2 & $160 \pm 4$ & $18,7 \pm 0,1$ & $30,9 \pm 0,5$ & $1,25 \pm 0,07$ & $20,3 \pm 0,2$ \\
\hline 4 & 21 & 22 & $\mathrm{CO}_{2}$ & 2 & $152 \pm 5$ & $18,8 \pm 0,1$ & $30,4 \pm 0,7$ & $1,10 \pm 0,03$ & $20,3 \pm 0,2$ \\
\hline 6 & 24 & 17 & $\mathrm{CO}_{2}$ & 6 & $222 \pm 2$ & $20,9 \pm 0,1$ & $25,6 \pm 0,6$ & $1,11 \pm 0,03$ & $21,0 \pm 0,1$ \\
\hline 6 & 26 & 17 & $\mathrm{CO}_{2}$ & 6 & $221 \pm 5$ & $23,0 \pm 0,1$ & $18,8 \pm 0,6$ & $1,14 \pm 0,13$ & $21,5 \pm 0,3$ \\
\hline 4 & 22 & 17 & $\mathrm{Ar}-15 \% \mathrm{CO}_{2}$ & 3 & $188 \pm 1$ & $19,7 \pm 0,0$ & $15,4 \pm 0,3$ & $1,08 \pm 0,08$ & $17,9 \pm 0,2$ \\
\hline 4 & 24 & 17 & $\mathrm{Ar}-15 \% \mathrm{CO}_{2}$ & 3 & $189 \pm 3$ & $21,6 \pm 0,0$ & $10,2 \pm 0,3$ & $1,10 \pm 0,02$ & $19,0 \pm 0,1$ \\
\hline 6 & 24 & 17 & $\mathrm{Ar}-15 \% \mathrm{CO}_{2}$ & 3 & $235 \pm 0$ & $20,8 \pm 0,0$ & $14,4 \pm 0,9$ & $1,11 \pm 0,04$ & $17,8 \pm 0,1$ \\
\hline 6 & 26 & 17 & $\mathrm{Ar}-15 \% \mathrm{CO}_{2}$ & 3 & $242 \pm 2$ & $22,7 \pm 0,0$ & $10,3 \pm 0,3$ & $1,10 \pm 0,05$ & $18,9 \pm 0,1$ \\
\hline
\end{tabular}




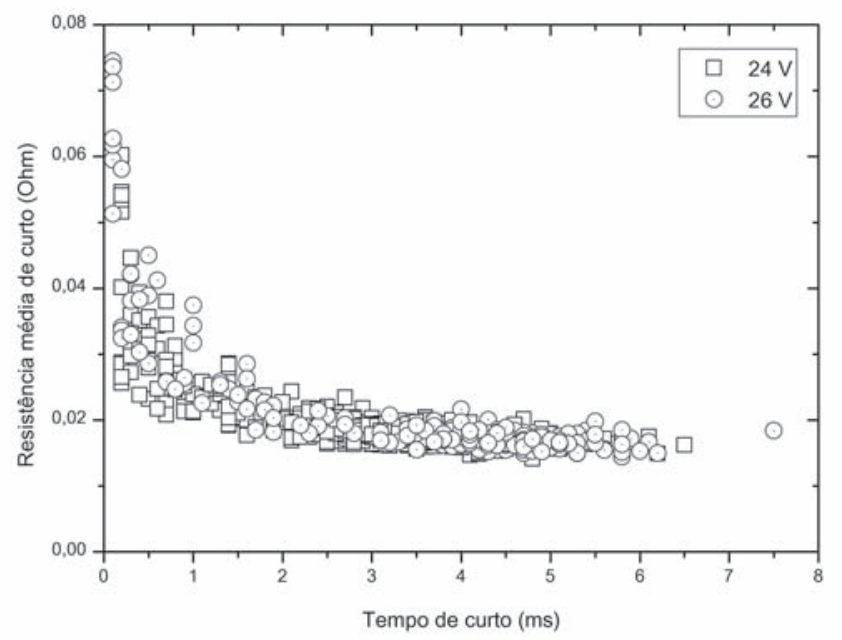

(a)

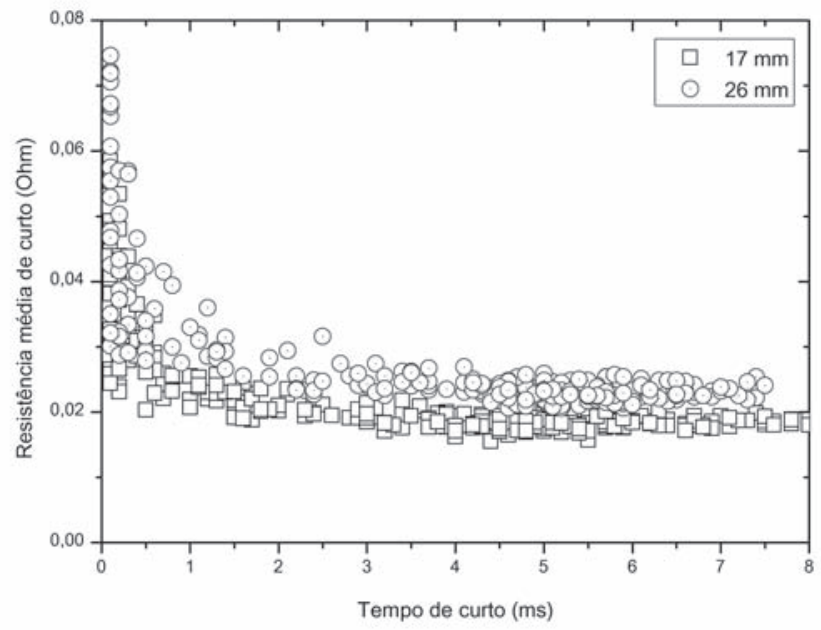

(b)

Figura 6. Variação do valor médio da resistência elétrica em cada curto em função de sua duração. Comparações entre dois testes com (a) velocidade de alimentação $(f)$ de $4 \mathrm{~m} / \mathrm{min}$ e dois níveis de tensão média de soldagem e (b) $f=6 \mathrm{~m} / \mathrm{min}$ e dois valores de $D B C P$.

A tabela 2 compara valores medidos de resistência durante o curto-circuito com a metodologia proposta (figura 4) com valores obtidos pela média da resistência média de cada curto levando em conta $\left[\left(\mathrm{R}_{\mathrm{cc}}\right)_{\text {Corte }=1}\right]$ no cálculo, ou não $\left[\left(\mathrm{R}_{\mathrm{cc}}\right)\right.$ Corte $=20$, os curtos-circuitos de menor duração (arbitrariamente considerados como aqueles com duração menor que $1 \mathrm{~ms}$ para a taxa de aquisição usada, $20 \mathrm{kHz}$ ). Os resultados indicam, dentro da faixa relativamente estreita de variação dos parâmetros, um efeito desprezível da velocidade de alimentação e da tensão de operação. Por outro lado, quando a resistência é obtida como a média dos valores médios de cada período de curto, observa-se uma clara tendência do resultado ser maior quando se considera os curtos-circuitos de menor duração. Além disto, quando curtos-circuitos de duração inferior a 1 ms são desconsiderados, os resultados ficam próximos dos obtidos pela média dos valores individuais de resistência abaixo de $40 \mathrm{mOhm}$.

Supondo que a maior resistência elétrica observada para os curtos-circuitos mais rápidos esteja associada com uma resistência de contato entre o eletrodo e a poça de fusão, uma estimativa do valor desta pode ser obtida através dos dados experimentais. Para isto determinou-se o valor da resistência do eletrodo para curtos-circuitos com duração inferior a $1 \mathrm{~ms}$, desprezando-se, contudo os mais rápidos (isto é, foram considerados curtos-circuitos com duração entre 2 e 19 pontos para a taxa de aquisição de dados de $20 \mathrm{kHz}$ ). A resistência de contato foi definida como a diferença entre o valor da resistência dos curtos-circuitos rápidos e dos longos (com duração superior a 20 pontos). A tabela 3 mostra alguns resultados destas estimativas:

Os resultados sugerem uma resistência de contato entre o eletrodo e a poça, durante curtos-circuitos de duração inferior a $1 \mathrm{~ms}$, entre cerca de 4 e $7 \mathrm{~m} \Omega$. Sugerem, também, uma possível redução desta resistência com o aumento da corrente de soldagem. Contudo, como o efeito destaresistência parece afetar de forma pouco significativa o cálculo da resistividade do eletrodo com a metodologia aqui adotada, ela não será mais considerada neste trabalho.

Tabela 2. Resistência de curto-circuito medida pela média de todos os pontos $\left[\left(R_{c c}\right)_{\text {Padrão }}\right]$ e pela média da resistência média de cada curto considerando-se ou não os curtos-circuitos de menor duração. Testes $\operatorname{com} f=4$ e $6 \mathrm{~m} / \mathrm{min}$, tensão ajustada de 22,24 e $26 \mathrm{~V}$, proteção de $\mathrm{Ar}-15 \% \mathrm{CO}_{2}$ e $D B C P=17 \mathrm{~mm}$.

\begin{tabular}{c|c|c|c|c}
\hline $\begin{array}{c}f \\
(\mathrm{~m} / \mathrm{min})\end{array}$ & Tensão ajustada $(\mathrm{V})$ & $\begin{array}{c}\left(R_{c}\right)_{\text {Padrão }} \\
(\mathrm{m} \Omega)\end{array}$ & $\begin{array}{c}\left(R_{c)}\right)_{\text {Corte }} \\
(\mathrm{m} \Omega)\end{array}$ & $\begin{array}{c}\left(R_{c c}\right)_{\text {Corte }}=20 \\
(\mathrm{~m} \Omega)\end{array}$ \\
\hline 4 & 22 & $16,4 \pm 3,0(18164)$ & $17,9 \pm 3,6(323)$ & $16,4 \pm 1,1(258)$ \\
\hline 4 & 24 & $16,2 \pm 2,8(12071)$ & $17,1 \pm 2,5(174)$ & $16,5 \pm 1,4(154)$ \\
\hline 6 & 24 & $16,9 \pm 3,4(17162)$ & $20,1 \pm 4,3(522)$ & $16,6 \pm 1,3(261)$ \\
\hline 6 & 26 & $16,8 \pm 3,5(12257)$ & $20,2 \pm 5,0(358)$ & $16,6 \pm 1,2(189)$ \\
\hline
\end{tabular}

Observações: Média \pm Desvio Padrão (Número de medidas)

$\left(\mathrm{R}_{\mathrm{cc}}\right)_{\text {Padrão }}$ - Média de todos os pontos abaixo de $40 \mathrm{~m} \Omega$ (ver figura 4)

$\left(R_{c c}\right)_{\text {Corte }=1}$ - Média das médias dos curtos desprezando curtos menores que $0,05 \mathrm{~ms}$

$\left(\mathrm{R}_{\mathrm{cc}}\right)_{\text {Corte }=20}$ - Média das médias dos curtos desprezando curtos menores que $1,0 \mathrm{~ms}$ 
Tabela 3. Resultados (média \pm desvio padrão) de alguns ensaios. $f$-Velocidade de alimentação, $U o$ - Tensão ajustada na fonte, $n-$ Número de réplicas na condição, $I$ e $U$ - Corrente e tensão médias de soldagem.

\begin{tabular}{c|c|c|c|c|c|c|c|c}
\hline $\begin{array}{c}f \\
(\mathrm{~m} / \mathrm{min})\end{array}$ & $\begin{array}{c}U o \\
(\mathrm{~V})\end{array}$ & $\begin{array}{c}D B C P \\
(\mathrm{~mm})\end{array}$ & Gás de Proteção & $n$ & $I(\mathrm{~A})$ & $U(\mathrm{~V})$ & $F c c(\%)$ & $\begin{array}{c}R_{p} \\
(\mathrm{~m} \Omega)\end{array}$ \\
\hline 4 & 22 & 17 & $\mathrm{Ar}-15 \% \mathrm{CO}_{2}$ & 3 & $188 \pm 1$ & $19,7 \pm 0,0$ & $15,4 \pm 0,3$ & $6,0 \pm 0,3$ \\
\hline 4 & 24 & 17 & $\mathrm{Ar}-15 \% \mathrm{CO}_{2}$ & 3 & $189 \pm 3$ & $21,6 \pm 0,0$ & $10,2 \pm 0,3$ & $6,0 \pm 0,7$ \\
\hline 6 & 24 & 17 & $\mathrm{Ar}-15 \% \mathrm{CO}_{2}$ & 3 & $235 \pm 0$ & $20,8 \pm 0,0$ & $14,4 \pm 0,9$ & $3,6 \pm 0,2$ \\
\hline 6 & 26 & 17 & $\mathrm{Ar}-15 \% \mathrm{CO}_{2}$ & 3 & $242 \pm 2$ & $22,7 \pm 0,0$ & $10,3 \pm 0,3$ & $4,2 \pm 0,3$ \\
\hline 4 & 21 & 14 & $\mathrm{CO}_{2}$ & 2 & $178 \pm 3$ & $18,4 \pm 0,0$ & $30,9 \pm 0,3$ & $7,4 \pm 0,3$ \\
\hline 4 & 21 & 18 & $\mathrm{CO}_{2}$ & 2 & $160 \pm 4$ & $18,7 \pm 0,1$ & $30,9 \pm 0,5$ & $7,2 \pm 0,5$ \\
\hline 4 & 21 & 22 & $\mathrm{CO}_{2}$ & 2 & $152 \pm 5$ & $18,8 \pm 0,1$ & $30,4 \pm 0,7$ & $6,9 \pm 0,0$ \\
\hline
\end{tabular}

Tabela 4. Resistividade corrigida e temperatura efetiva do eletrodo considerando valores de literatura para a resistência de contato entre o eletrodo e o bico de contato.

\begin{tabular}{c|c|c|c|c|c|c|c|c}
\hline \multirow{2}{*}{$\begin{array}{c}D B C P \\
(\mathrm{~mm})\end{array}$} & \multicolumn{2}{|c|}{$\mathrm{R}_{\mathrm{c}}=1,5 \mathrm{~m} \Omega[4,10]$} & \multicolumn{2}{c|}{$\mathrm{R}_{\mathrm{c}}=3,4 \mathrm{~m} \Omega[11]$} & \multicolumn{2}{c|}{$\mathrm{R}_{\mathrm{c}}=14 \mathrm{~m} \Omega[12]$} & \multicolumn{2}{c}{$\mathrm{R}_{\mathrm{c}}=60 \mathrm{~m} \Omega[12]$} \\
\cline { 2 - 10 } & $\rho_{\mathrm{e}}(\mu \Omega \cdot \mathrm{m})$ & $\mathrm{T}_{\mathrm{ef}}\left({ }^{\circ} \mathrm{C}\right)$ & $\rho_{\mathrm{e}}(\mu \Omega . \mathrm{m})$ & $\mathrm{T}_{\text {ef }}\left({ }^{\circ} \mathrm{C}\right)$ & $\rho_{\mathrm{e}}(\mu \Omega . \mathrm{m})$ & $\mathrm{T}_{\text {ef }}\left({ }^{\circ} \mathrm{C}\right)$ & $\rho_{\mathrm{e}}(\mu \Omega \cdot \mathrm{m})$ & $\mathrm{T}_{\text {ef }}\left({ }^{\circ} \mathrm{C}\right)$ \\
\hline 14 & 1,21 & 1450 & 1,05 & 800 & 0,20 & $<25$ & $<0$ & -- \\
\hline 18 & 1,15 & 950 & 1,03 & 770 & 0,37 & 270 & $<0$ & -- \\
\hline 22 & 1,02 & 770 & 0,93 & 700 & 0,38 & 270 & $<0$ & -- \\
\hline
\end{tabular}

A tabela 4 mostra valores da resistividade do eletrodo calculados (equação 3) levando em conta valores da resistência de contato entre o eletrodo e o bico dados pela literatura $[4,10-$ 12]. Os dados mostrados correspondem às condições das três primeiras linhas da tabela 1 . São também mostradas estimativas da temperatura efetiva do eletrodo para esses valores de resistividade com base na curva da figura 2 para o aço SAE 1020. Os valores de temperatura efetiva parecem muito elevados quando os dados de resistência de contato de Terasaki\& Simpson [4] (baseados em resultados de Huismann[10]) são usados e muito baixos para os valores sugeridos por Ersoy e Colaboradores [12]. As temperaturas estimadas com o valor da resistência de contato proposto por Vilarinho [11] parecem mais próximas do esperado embora, possivelmente, essas temperaturas estejam também elevadas. Deve-se ressaltar, contudo, que (1) a curva de resistividade do aço SAE 1020 não corresponde exatamente à do aço do eletrodo ER70S6 (que apresenta teores mais elevados de $\mathrm{Mn}$ e $\mathrm{Si}$ ) e que (2) a resistência de contato deve variar com as condições de soldagem e, mais importante, com as condições superficiais do arame e do bico de contato. Medidas da resistividade em cinco arames de corridas diferentes desta classe de eletrodoindicam uma resistividade, a $25^{\circ} \mathrm{C}$, de $0,305 \pm 0,005 \mu \Omega$.m, cerca de $50 \%$ maior do que a sugerida pela figura 2. Além disto, medidas de resistividade a alta temperatura mostram valores de resistividade maiores, em cerca de $20-30 \%$, particularmente para a faixa de temperatura em que o aço não foi ainda austenitizado. Assim, temperaturas efetivas do eletrodo menores podem ser esperadas.

Considerando o esquema da figura 1 e desprezando $R_{p}$, valor médio de $\mathrm{R}_{\mathrm{cc}}$, de um número suficientemente grande de testes, pode ser representado, em uma primeira aproximação, como:

$$
\bar{R}_{c C}=\bar{R}_{C}+\frac{\bar{R}_{\varepsilon}}{A} \cdot s \bar{R}_{c C}=\bar{R}_{C}+\frac{\bar{R}_{\varepsilon}}{A} \cdot S
$$

A figura 7 mostra a relação entre os valores médios, para cada distância do bico de contato à peça, da resistência de curtocircuito em função dessa distância para um total de 127 testes realizados em diferentes condições. A reta obtida por regressão para estes dados, comparando-a com a equação (13), sugere uma resistência entre o bico e o eletrodo de $8,1 \pm 1,0 \mathrm{~m} \Omega$ e uma resistividade média deste eletrodo de 0,62 $\pm 0,06 \mu \Omega$.m. Utilizando o mesmo procedimento para um conjunto de 50 testes feitos em outro trabalho [19] com um arame da mesma classe e diâmetro, mas usando uma fonte convencional eletromagnética, distância do bico de contato à peça de 15,20 e $25 \mathrm{~mm}$, velocidades de alimentação do arame de 2,4 e $6 \mathrm{~m} / \mathrm{min}$ e proteção de $\mathrm{CO}_{2}$, permitem obter a equação (14)com coeficientes similares:

$$
\bar{R}_{c c}=(7,4 \pm 1,0)+(0,54 \pm 0,05) \cdot s
$$

Assim, estes resultados sugerem, para os dois conjuntos de testes considerados, uma resistividade média do eletrodo entre aproximadamente $0,55 \mathrm{e} 0,65 \mu \Omega . \mathrm{m}$ e uma resistência elétrica entre o bico e a peça entre 6 e $9 \mathrm{~m} \Omega$. Estes últimos valores estão entre aqueles obtidos por Vilarinho [11] e os menores valores encontrados por Ersoy e Colaboradores [12]. Além disto, considerando a análise feita anteriormente e os valores 
de resistividade elétrica da figura2, estes valores correspondem a uma temperatura efetiva do eletrodo razoável,entre aproximadamente 400 e $500^{\circ} \mathrm{C}$.

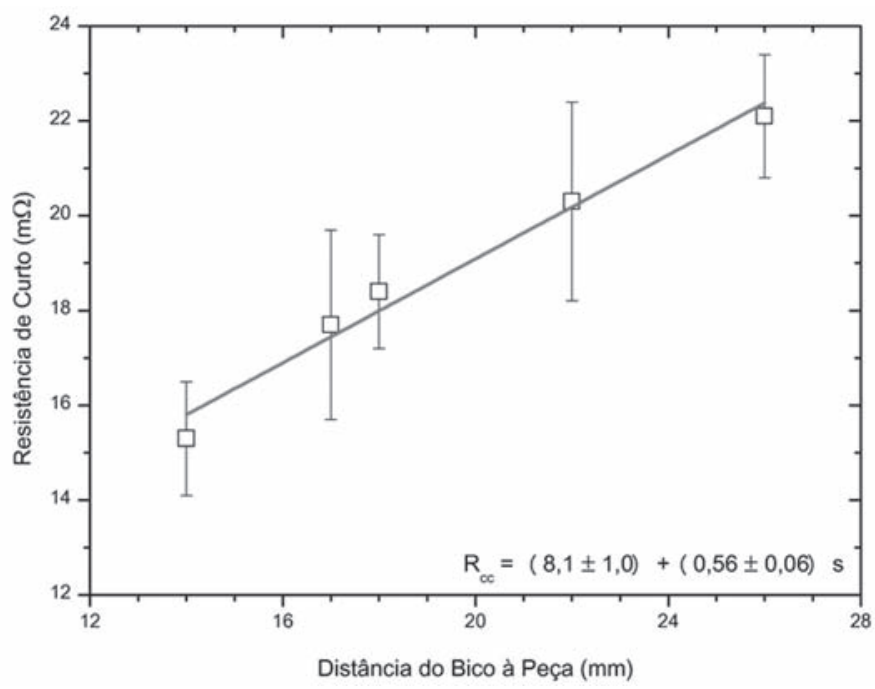

Figura 7. Relação entre a resistência média de curto-circuito e a distância entre o bico de contato e a peça. Cada média engloba testes com diferentes velocidades de alimentação de arame (4 e $6 \mathrm{~m} / \mathrm{min})$, tensões de operação e gases de proteção $\left(\mathrm{CO}_{2}\right.$ e $\mathrm{Ar}-$ $\left.15 \% \mathrm{CO}_{2}\right)$. Equação de regressão com os coeficientes \pm o seu erro padrão.

\subsection{Soma das quedas anódica e catódica $\left(\mathrm{U}_{\mathrm{AC}}\right)$}

A figura 8 mostra detalhes de curtos-circuitos medidos com diferentes taxas de aquisição. Estes dados foram retirados da biblioteca de sinais de soldagem de um dos autores e se referem a testes com arame ER70S6 de 1,2 $\mathrm{mm}$ de diâmetro, $f=4 \mathrm{~m} / \mathrm{min}$, $D B C P=19 \mathrm{~mm}$ e proteção de $\mathrm{Ar}-2 \% \mathrm{CO}_{2}$ coletados com taxas de aquisição de dados de 5 a $80 \mathrm{kHz}$. Nesta figura, os pontos cheios correspondem àqueles que seriam usados para a determinação de $U_{A C}$.

Observou-se que, para taxas de aquisição de dados acima de 10 ou $20 \mathrm{kHz}$, dados são medidos com frequência na condição de transiçãoentre a operação com arco e em curto-circuito. A técnica aqui adotada, através do cálculo da diferença entre dois pontos não imediatamente adjacentes (equação 7), permite estimar a diferença de tensão entre essas duas condições de operação desde que exista, no máximo, um ponto intermediário. Para as taxas mais elevadas de aquisição de dados, observouse uma maior dispersão dos resultados possivelmente uma conseqüência do chaveamento da máquina de soldagem usada.

$\mathrm{O}$ efeito de um fator adicional avaliado sobre os resultados de $U_{A C}$ foi a duração dos curtos-circuitos (figura 9). Neste caso, ao contrário do observado na determinação da resistividade do arame, não foi observada uma influência forte deste tempo, exceto para curtos-circuitos extremamente rápidos cuja forma é distorcida durante a aquisição de dados.

Para reduzir o efeito dos curtos-circuitos rápidos e reduzir a dispersão dos resultados nas medidas de $\mathrm{U}_{\mathrm{AC}}$, foi elaborada uma metodologia mais sofisticada para a determinação deste parâmetro, baseada na diferença das médias de um número de
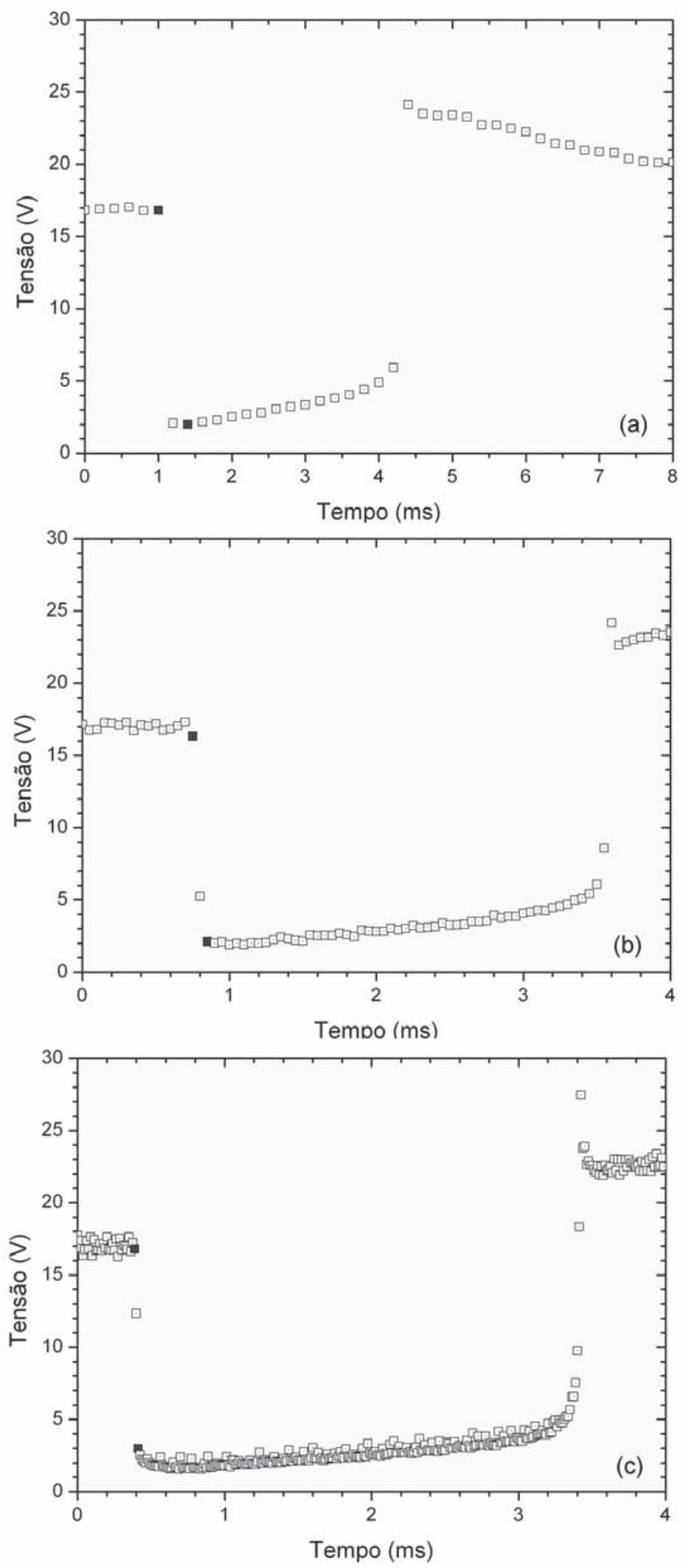

Figura 8. Detalhes do instante de iniciação de um curto-circuito em testes realizados com condições similares e monitorados com diferentes taxas de aquisição de dados: (a) $5 \mathrm{kHz}$, (b) $20 \mathrm{kHz}$ e (c) $80 \mathrm{kHz}$. Os pontos cheios correspondem àqueles que seriam usados pelo programa Sinal para a determinação de $U_{A C}$ (ver seção 3.2). 


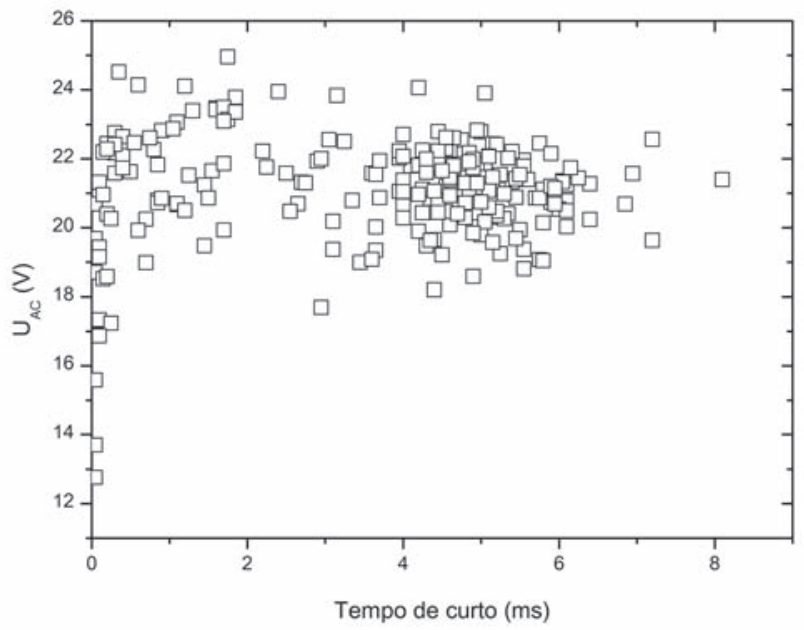

Figura 9. Variação do valor de $U_{A C}$ com a duração do período de curto-circuito durante um teste de soldagem.

pontos imediatamente antes e imediatamente após o início do curto-circuito, isto é:

$$
\Delta U(i)=\operatorname{Media}(i+N, M)-\operatorname{Média}(i, P)
$$

Onde Media $(i+N, M)$ e Media $(i, P)$ são, respectivamente, as médias de $M$ valores de tensão $\left(U_{i+N}, U_{i+N+}, U_{i+N+M}\right)$ medidos após o início do curto e de $P$ valores $\left(U_{i}, U_{i-1}, U_{i-P}\right)$ medidos antes de seu início. Para as condições de aquisição de dados usadas neste trabalho (frequências de aquisição de 10 e $20 \mathrm{kHz}$ ), os valores de $N, M$ e $P$ foram definidos, após uma avaliação inicial, como, respectivamente, 2, 3 e 3 . Adotou-se, ainda, como condição para que a diferença fosse válida, que dois pontos consecutivos usados no cálculo das médias não diferissem por mais de $4 \mathrm{~V}$ (valor definido de forma arbitrária). Esta última condição exclui das diferenças calculadas aquelas associadas com curtos-circuitos ou períodos de arco muito rápidos.

A figura 10 mostra a dispersão das medidas individuais de $U_{A C}$ para um mesmo teste de soldagem usando as duas abordagens para o cálculo deste parâmetro. Pode-se observar, para o uso do procedimento baseado na equação (15), uma menor dispersão dos valores medidos, particularmente a ausência de valores menores, possivelmente ligados a curtos-circuitos rápidos.

Finalmente, embora o foco deste trabalho não tenha sido uma análise mais profunda do efeito das diferentes variáveis do processo nos parâmetros estudados, a figura 11 mostra os valores medidos de $U_{A C}$ para todos os testes feitos em função do gás de proteção, incluindo testes com proteção de $\mathrm{Ar}-2 \% \mathrm{O}_{2}$ feitos anteriormente. Estes resultados foram obtidos para diferentes valores de velocidade de alimentação, $D B C P$ e tensão ajustada na fonte. Percebe-se o efeito significativo da composição do gás, particularmente o seu teor de $\mathrm{CO}_{2}$, no valor deste parâmetro. Este gás tem um efeito oxidante na atmosfera do arco (devido à sua decomposição a alta temperatura) e tende a aumentar as perdas térmicas do arco para o meio ambiente. Além disto, como uma conseqüência direta deste último efeito, o arco tende a se contrair, interagindo com o eletrodo em uma área mais restrita. A variação observada de $U_{A C}$ pode estar relacionada com este efeito, mas isto obviamente ainda demanda um trabalho adicional para a sua verificação.

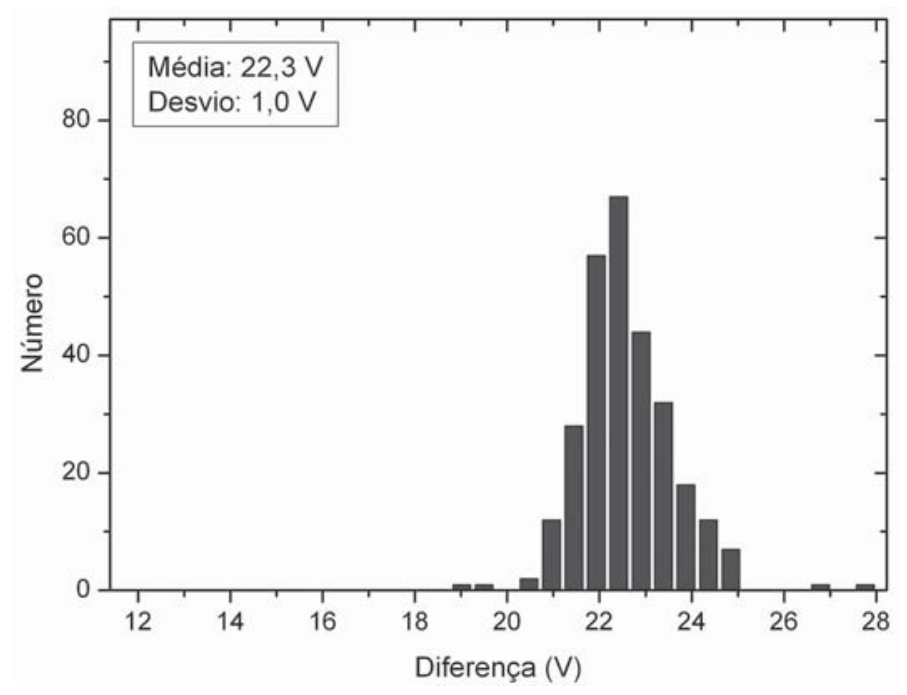

(b)

Figura 10. Distribuição de valores de $U_{A C}$ obtidos no mesmo teste usando as equações (12) (a) e (13) (b). Distância do bico de contato à peça $=17 \mathrm{~mm}$, velocidade de alimentação $=6 \mathrm{~m} / \mathrm{min}$, tensão regulada $=26 \mathrm{~V}$, velocidade de soldagem $=0,60 \mathrm{~m} / \mathrm{min}$ e proteção com 


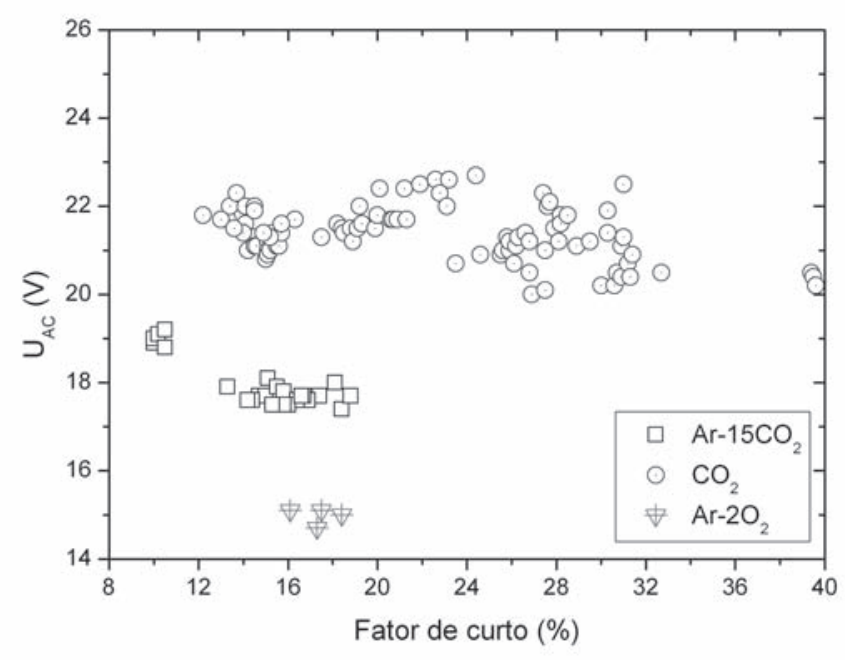

Figura 11. Valores medidos de $U_{A C}$ para dois diferentes gases de proteção.

\section{Conclusões}

O presente trabalho apresenta e avalia metodologias para a determinação de alguns parâmetros básicos para a modelagem matemática do processo de soldagem GMAW através da análise direta dos sinais de corrente e tensão de soldagem. Mostrou-se que:

- O valor médio da resistência elétrica durante um curtocircuito tende a cair com o aumento de sua duração. Para curtoscircuitos maiores que cerca de $1 \mathrm{~ms}$, o valor desta resistência tende a se estabilizar.

- Para a determinação da resistividade elétrica do eletrodo em condições de soldagem com transferência por curto-circuito é importante considerar a existência da resistência de contato entre este e o bico de contato. O presente trabalho desenvolve uma metodologia para avaliar o valor desta resistência baseada $\mathrm{em}$ testes experimentais de soldagem. Esta metodologia permitiu obter valores para esta resistência entre 6 e $9 \mathrm{~m} \Omega$. Para arames de aço carbono (AWS ER 70S-6) de 1,2 mm de diâmetro, a resistividade elétrica durante a soldagem, nas condições testadas, vale entre aproximadamente 0,55 e $0,65 \mu \Omega$.m.

- Os resultados obtidos indicam que, em curtos-circuitos de pequena duração (inferior a $1 \mathrm{~ms}$ ), existe uma resistência de contato entre o eletrodo e a poça com um valor entre cerca de 4 e $7 \mathrm{~m} \Omega$ (quando comparado com curtos-circuitos de maior duração).

- A metodologia usada para a determinação da soma das quedas de tensão anódica e catódica $\left(U_{A C}\right)$ depende da taxa de aquisição de dados utilizada, tendendo o valor de $\mathrm{U}_{\mathrm{AC}}$ a diminuir quando taxas de aquisição elevadas (próximas ou superiores a $10^{5} \mathrm{~Hz}$ ) são usadas. Para as taxas de aquisição usadas neste trabalho (10 e $20 \mathrm{kHz}$ ) e menores (até $2,5 \mathrm{kHz}$ ), a metodologia usada (particularmente o intervalo de pontos para a determinação de $U_{A C}$ ) fornece valores similares de $U_{A C}$.

- A soma das quedas de tensão anódica e catódica depende da composição do gás de proteção, tendendo a aumentar com o teor de $\mathrm{CO}_{2}$.

\section{Referências}

[1] SHAHI, A. S., PANDEY, S. Modelling of the Effects of Welding Conditions on Dilution of Stainless Steel Claddings Produced by Gas Metal Arc Welding Procedures, Journal of Materials Processing Technology 196 (1-3) 339-344, 2008.

[2] FAN, H. G., KOVACEVIC, R. Droplet Formation, Detachment, and Impingement on the Molten Pool in Gas Metal Arc Welding, Metal. Mat. Transactions B 30 (4) 791-801, 1997. [3] ZHAO, Y., ZHOU, H., SHI, Y. The Study of Surface Active Element on Weld Pool Development in A-TIG Welding, ModellingSimul.Mater.Sci. Eng. 14 331-349, 2006.

[4] TERASAKI, H.; SIMPSON, S. W. Circuit simulation for gas metal arc welding system. Science and Technology of Welding and Joining, p.121-124, 2005.

[5] REED-HILL, R. E.; ABBASCHIAN, R.; ABBASCHIAN, L. Physical metallurgy principles. Boston: Editora PWS, Kent Publishing Company, 1999. 914p.

[6] HALlidAY, D.; RESNICK, R.; WALKER, J. Fundamentals of physics. $8^{a}$, Danvers: John Wiley \& Sons, 2008. 1248p.

[7] LIDE, DAVID R. Handbook of Chemistry and Physics Special Student Edition. CRC Press, 1991-1992.

[8] SCOTTI, A.; PONOMAREV, V.; RESENDE, A. The influence of the electrode materials and shielding gas mixture on the specific electric resistances of the drop/column of the arc in MIG/MAG welding. IIW Doc. XII-1909-06. 9p.

[9] KIM, Y. S.; MCELIGOT D. M.; EAGAR T. W. Analyses of electrode heat transfer in gas metal arc welding. Welding Journal, p. 20-31, 1991.

[10] HUISMANN, G. : Contact resistance in MIG welding, IIW Doc. 212-950-99, 1999.

[11] VILARINHO, L. Pesquisa não publicada, dados fornecidos através de contato pessoal. 2010.

[12] U. ERSOY; S. J. HU, AND E. KANNATEY-ASIBU. Observation of arc start instability and spatter generation in GMAW. Welding Journal, v.87, p. 51-56, Feb. 2008.

[13] BLESS, S. J. Drop transfer in short circuit welding, J. of Phys. D: Appl. Phys. 7, p. 526-539, 1974.

[14] LANCASTER, J. F. The physics of welding. 2a, Oxford: Pergamon Press, 1986. 340p.

[15] GUILE, A. E. Arc cathode and anode phenomena. Annual Assembly of the International Institute of Welding, 1970.

[16] SCOTTI, A.; PONOMAREV, V.; COSTA. A. V. A methodology for voltage drop determination in GMA welding: arcs with short-circuiting metal transfer. Eur. Phys. J. Appl. Phys. n. 34, p. 231-236, 2006.

[17] MODENESI, P. J.; REIS, R. I.. A model for melting rate phenomena in GMA welding. Journal of Materials Processing Technology, p. 199-205, 2007.

[18] JILONG MA, APPS, R. L., MIG transfer discovery of importance to industry. Weld. \& Metal Fab., p. 168-175, 1982.

[19] DEIRÓ, W. C. Monitoração e Controle em Soldagem, Desenvolvimento de Sistemas de Monitoração, Relatório de Iniciação Científica, Universidade Federal de Minas Gerais, $17 \mathrm{p} ., 2004$. 